7 Zhang W, Chen Y, Wei H, et al. Antiapoptotic activity of autocrine interleukin-22 and therapeutic effects of interleukin-22-small interfering RNA on human lung cancer xenografts. Clin Cancer Res 2008; 14: 6432-6439.

8 Dugger DT, Gerriets JE, Miller LA. Attenuated airway epithelial cell interleukin-22R1 expression in the infant nonhuman primate lung. Am J Respir Cell Mol Biol 2015; 53: 761-768.

9 Guillon A, Jouan Y, Brea D, et al. Neutrophil proteases alter the interleukin-22-receptor-dependent lung antimicrobial defence. Eur Respir J 2015; 46: 771-782.

10 Sonnenberg GF, Fouser LA, Artis D. Border patrol: regulation of immunity, inflammation and tissue homeostasis at barrier surfaces by IL-22. Nat Immunol 2011; 12: 383-390.

11 Chen Z, Fillmore CM, Hammerman PS, et al. Non-small-cell lung cancers: a heterogeneous set of diseases. Nat Rev Cancer 2014; 14: 535-546.

12 Camp RL, Dolled-Filhart M, Rimm DL. X-tile: a new bio-informatics tool for biomarker assessment and outcomebased cut-point optimization. Clin Cancer Res 2004; 10: 7252-7259.

13 Győrffy B, Surowiak P, Budczies J, et al. Online survival analysis software to assess the prognostic value of biomarkers using transcriptomic data in non-small-cell lung cancer. PLoS One 2013; 8: e82241.

14 Mizuno H, Kitada K, Nakai K, et al. PrognoScan: a new database for meta-analysis of the prognostic value of genes. BMC Med Genomics 2009; 2: 18.

15 Goswami CP, Nakshatri H. PROGgeneV2: enhancements on the existing database. BMC Cancer 2014; 14: 970.

16 Aguirre-Gamboa R, Gomez-Rueda H, Martínez-Ledesma E, et al. SurvExpress: an online biomarker validation tool and database for cancer gene expression data using survival analysis. PLoS One 2013; 8: e74250.

\title{
Idelalisib-related pneumonitis
}

\author{
To the Editor:
}

Idelalisib (Zydelig; Gilead Sciences Inc., Foster City, CA, USA) has recently been approved in combination with rituximab for the treatment of refractory chronic lymphocytic leukaemia (CLL) in patients who have received at least one prior therapy, as a first-line treatment in the presence of a 17p deletion or TP53 mutation in patients unsuitable for chemoimmunotherapy, or as a monotherapy in follicular lymphoma refractory to two prior lines of treatment. Idelalisib specifically inhibits phosphatidylinositol 3-kinase $\delta$ (PI3K $\delta$ ), which is part of the B-cell receptor signalling pathway, the activity of which is increased in B-cell malignancies, and which promotes cellular proliferation and survival [1-4]. Here, we report on five patients who were diagnosed with pneumonitis related to idelalisib treatment.

The patients were three men and two women with a median age of 72 years (interquartile range 68-79 years). None had a history of respiratory disease or environmental exposure and none was a current smoker (one had a 7- and another, a 10-pack-year history of smoking). The patients had received idelalisib for a mean time of 7.8 months (range 2-16 years). Four of them were being treated for refractory CLL whereas one had progressive lymphoplasmacytic lymphoma in the setting of Waldenström disease. All patients were receiving idelalisib at a dose of $150 \mathrm{mg}$ twice daily, except for one patient who had been receiving $100 \mathrm{mg}$ twice daily for 14 months (the initial dosing of $150 \mathrm{mg}$ twice daily had to be decreased 2 months after introduction for severe hypertriglyceridaemia). The mean number of previous lines of therapy was 3.8 (range 1-5). For three patients with CLL, idelalisib was initially administered with ofatumumab. At the time of pneumonitis, ofatumumab had been stopped 4-12 months previously. The fourth patient with CLL was receiving rituximab in combination with idelalisib. The patient with Waldenström disease received idelalisib alone. At the time of pneumonitis, three patients had controlled haematological disease, whereas two patients had progressive disease.

All patients complained of cough, dyspnoea and fever. Four had progression of their clinical symptoms over 3-9 weeks, whereas one had progression over 5 days. Four patients had crackles on physical examination and one had a normal examination. One patient had a severe skin reaction attributed to idelalisib toxicity. One patient had diarrhoea.

A lung computed tomography (CT) scan showed diffuse ground-glass opacities $(n=3)$, consolidations $(\mathrm{n}=2)$, diffuse micronodules $(\mathrm{n}=1)$ and pleural effusions $(\mathrm{n}=2)$ (figure 1$)$.

All patients except one required oxygen supplementation and admission to an intensive care unit. Two of these patients required mechanical ventilation. Three of the patients had a bronchoalveolar lavage (BAL). The mean \pm SD total cell count was $583 \pm 380$ per $\mu \mathrm{L}$. Lymphocytic alveolitis was found in two patients (mean lymphocyte proportion $71 \pm 5 \%$ ), whereas one patient had neutrophilic alveolitis (77\% neutrophils, $1 \%$ lymphocytes). An extensive search for pathogens, including respiratory viruses, bacteria and fungi, was 
negative in the BAL, except in one patient in whom Stenotrophomonas maltophilia was found to be associated with lymphocytic alveolitis (total cell count 980 per $\mu \mathrm{L} ; 66 \%$ lymphocytes, $1 \%$ neutrophils). The presence of $S$. maltophilia was considered a bronchial colonisation. Because of respiratory impairment, two patients could not have a BAL. They both had noninvasive microbiological investigations. Sputum examinations for bacteria and fungi, including Pneumocystis jiroveci, were negative. Nasal aspirates found rhinovirus in patients in whom the clinical picture could not be explained by this finding.

All patients received broad-spectrum antibiotics with no significant efficacy. Idelalisib was definitively stopped in all patients. Four received steroids (prednisone $1 \mathrm{mg} \cdot \mathrm{kg}^{-1}$ of body weight per day for three, $0.5 \mathrm{mg} \cdot \mathrm{kg}^{-1}$ for one), among whom two received three pulses of $500 \mathrm{mg}$ methylprednisolone beforehand. The median time of the follow-up was 157 days (range 12-267 days). Among patients who received corticosteroids, three had a resolution of pneumonitis and one patient died 12 days after admission due to multiorgan failure. The patient with S. maltophilia did not receive any targeted antibiotics and had a favourable outcome with steroids. The patient who was not treated with steroids had a favourable outcome after the interruption of idelalisib.

Herein, we report for the first time the clinical features of idelalisib-related pneumonitis. The median age of our patients was 72 years, similarly to the median age of patients who are diagnosed with CLL [5]. Respiratory symptoms, including cough, dyspnoea and pneumonia, together with digestive symptoms, were the most frequent adverse events reported in previously conducted clinical trials evaluating the efficacy of idelalisib.

It is very difficult to accurately assess the prevalence of idelalisib-induced pneumonitis, especially because the drug was approved very recently. In phase 1-3 studies, cough was reported in $12-29 \%$ of patients and dyspnoea in 10-18\%, whereas pneumonia was reported in $6-22 \%$ of patients [6-9]. Noninfectious pneumonitis was specifically diagnosed in $2-5 \%$ of patients treated with idelalisib, including fatal cases [7-10]. Among the five patients in our series, four patients (including two enrolled in a clinical trial) were treated in Hospital Saint Louis, Paris, France, which is mostly dedicated to onco-haematological patients. At the date of December 1, 2015, at this site, a total of 21 patients have been treated with idelalisib including those enrolled in clinical trials (i.e. 19\% were diagnosed with an idelalisib-related pneumonitis). At the same time, seven cases of idelalisib-related pneumonitis were declared in the French pharmacovigilance database (four being concomitantly treated with rituximab). In parallel, approximately 1000 patients have been treated with idelalisib in France since this drug was approved (data obtained from Gilead Sciences Inc.), leading to an estimation of the prevalence of idelalisib-associated pneumonitis of $0.7 \%$. However, these data should be taken with caution as they cannot be exhaustive and do not include patients treated in pre-licensing trials.
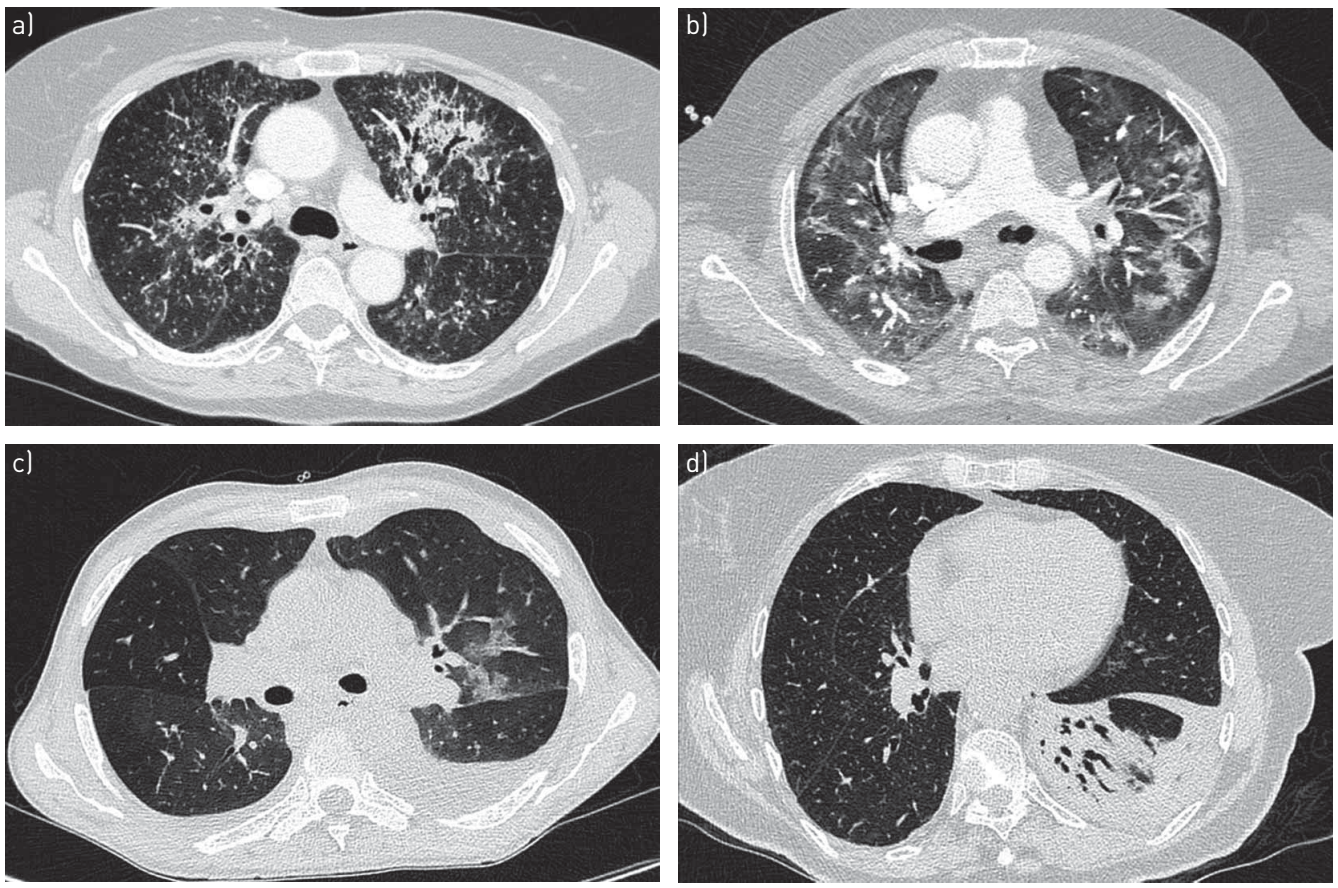

FIGURE 1 Lung CT scan patterns from patients with idelalisib-related pneumonitis showing ground-glass opacities a) with or b) without micronodules, associated with c) a pleural effusion or d) consolidation with a pleural effusion. 
Although rarely, rituximab has also been associated with lung toxicity [11]. One could postulate that pneumonitis could be related to rituximab rather than idelalisib when both are administered. However, pneumonitis has also been reported in clinical trials evaluating idelalisib as a monotherapy [7, 9]. In our series, only one patient received rituximab together with idelalisib. Although this patient had previously been treated with rituximab 2 years before with no related adverse events, we cannot firmly exclude a pulmonary toxicity of rituximab.

Currently, there are no data on the mechanisms of idelalisib-related pneumonitis. No lung biopsies were available for our patients. Two patients had lymphocytic alveolitis in BAL whereas one had neutrophilic alveolitis. The significance of this BAL finding as regards the mechanisms for pulmonary reaction to drugs is as yet unclear, inasmuch as a given drug may produce varied BAL changes amongst patients. In the lung CT scan patterns of our series, ground-glass opacities are consistent with hypersensitivity pneumonitis, although the consolidation pattern is rather consistent with organising pneumonia. Interestingly, both hypersensitivity pneumonitis and organising pneumonia have been reported as adverse events of mammalian target of rapamycin (mTOR) inhibitors such as everolimus, temsirolimus or sirolimus [12-15]. By inhibiting PI3K $\delta$, idelalisib also inhibits the mTOR pathway [10]. Thus, whether both idelalisib and mTOR inhibitor-related pneumonitis may share a similar pathophysiology remains to be explored [10].

Both lung physicians and haematologists should be aware of the possibility of drug-related lung manifestations in patients receiving idelalisib. Due to the potential severity of idelalisib-related pneumonitis, any respiratory symptom reported should be investigated. If pneumonia is suspected, the drug should be promptly discontinued. Because patients treated with idelalisib are usually severely immunocompromised, particularly those with refractory CLL, antimicrobial treatments should be introduced, and an extensive search for pathogens should be conducted before prior to diagnosing a patient with idelalisib-related pneumonitis. After infectious pneumonia has been investigated and the severity of the pneumonitis has been determined, steroids should be discussed. We did not reintroduce idelalisib in any of our patients. Thus, we cannot conclude that it could be an option, even at a lower dose, in cases of the unavailability of an alternative treatment for a haematological disease. In this case, a combination of idelalisib with low-dose steroids could be discussed.

0 @ERSpublications

Lung physicians and haematologists should be aware of the possibility of idelalisib-related lung manifestations http://ow.ly/XmjGm

Eglantine Haustraete ${ }^{1}$, Julie Obert ${ }^{1}$, Samia Diab $^{2}$, Sarah $\mathrm{Abbes}^{3}$, Jean-Marc Zini ${ }^{4}$, Sandrine Valade ${ }^{5}$, Nathalie Lerolle ${ }^{6}$, Nicolas Albin ${ }^{7}$, Bertrand Arnulf $^{8}$, Jean-David Bouaziz ${ }^{9}$, Claire Hussenet ${ }^{1}$, Abdellatif Tazi ${ }^{1,10}$ and Anne Bergeron 1,10

${ }^{1}$ AP-HP, Hôpital Saint-Louis, Service de Pneumologie, Paris, France. ${ }^{2}$ Groupe Médical Mounier, Pneumologie, Grenoble, France. ${ }^{3} \mathrm{CHU}$ Nantes, Service de Pneumologie, Nantes, France. ${ }^{4}$ AP-HP, Hôpital Saint-Louis, Service des Maladies du sang, Paris, France. ${ }^{5}$ AP-HP, Hôpital Saint-Louis, Service de Réanimation médicale, Paris, France. ${ }^{6}$ AP-HP, Hôpital Saint-Louis, Service de Maladies infectieuses, Paris, France. ${ }^{7}$ Institut Daniel Hollard, Service d'HématologieCancérologie, Grenoble, France. ${ }^{8}$ AP-HP, Hôpital Saint-Louis, Département d'Immuno-Hématologie, Paris, France. ${ }^{9}$ AP-HP, Hôpital Saint-Louis, Service de Dermatologie, Paris, France. ${ }^{10}$ Univ Paris Diderot, Sorbonne Paris Cité, U1153 CRESS, Biostatistics and Clinical Epidemiology research team, Paris, France.

Correspondence: Anne Bergeron, Service de Pneumologie, Hôpital Saint-Louis, 1, avenue Claude Vellefaux, 75475, Paris cedex 10, France. E-mail: anne.bergeron-lafaurie@aphp.fr

Received: Oct 092015 | Accepted after revision: Jan 03 2016 | First published online: Feb 252016

Conflict of interest: Disclosures can be found alongside the online version of this article at erj.ersjournals.com

\section{References}

1 Robak T, Robak P. BCR signaling in chronic lymphocytic leukemia and related inhibitors currently in clinical studies. Int Rev Immunol 2013; 32: 358-376.

2 Wiestner A. Emerging role of kinase-targeted strategies in chronic lymphocytic leukemia. Blood 2012; 120 : 4684-4691.

3 Shah A, Mangaonkar A. Idelalisib: a novel PI3K inhibitor for chronic lymphocytic leukemia. Ann Pharmacother 2015; 49: 1162-1170.

4 Yang Q, Modi P, Newcomb T, et al. Idelalisib: first-in-class PI3K delta inhibitor for the treatment of chronic lymphocytic leukemia, small lymphocytic leukemia, and follicular lymphoma. Clin Cancer Res 2015; 21: 1537-1542.

5 Heraly B, Morrison VA. How I treat chronic lymphocytic leukemia in older patients. J Geriatr Oncol 2015; 6: 333-340.

6 Kahl BS, Spurgeon SE, Furman RR, et al. A phase 1 study of the PI3K $\delta$ inhibitor idelalisib in patients with relapsed/refractory mantle cell lymphoma (MCL). Blood 2014; 123: 3398-3405.

7 Brown JR, Byrd JC, Coutre SE, et al. Idelalisib, an inhibitor of phosphatidylinositol 3-kinase p1108, for relapsed/ refractory chronic lymphocytic leukemia. Blood 2014; 123: 3390-3397. 
8 Furman RR, Sharman JP, Coutre SE, et al. Idelalisib and rituximab in relapsed chronic lymphocytic leukemia. N Engl J Med 2014; 370: 997-1007.

9 Gopal AK, Kahl BS, de Vos S, et al. PI3K $\delta$ inhibition by Idelalisib in patients with relapsed indolent lymphoma. N Engl J Med 2014; 370: 1008-1018.

10 Coutre SE, Barrientos JC, Brown JR, et al. Management of adverse events associated with idelalisib treatment: expert panel opinion. Leuk Lymphoma 2015; 1-8.

11 Hadjinicolaou AV, Nisar MK, Parfrey H, et al. Non-infectious pulmonary toxicity of rituximab: a systematic review. Rheumatology 2012; 51: 653-662.

12 Champion L, Stern M, Israël-Biet D, et al. Brief communication: sirolimus-associated pneumonitis: 24 cases in renal transplant recipients. Ann Intern Med 2006; 144: 505-509.

13 Duran I, Siu LL, Oza AM, et al. Characterisation of the lung toxicity of the cell cycle inhibitor temsirolimus. Eur J Cancer 2006; 42: 1875-1880

14 Kaplan B, Qazi Y, Wellen JR. Strategies for the management of adverse events associated with mTOR inhibitors. Transplant Rev 2014; 28: 126-133.

15 Wang WL, Yu LX. Acute respiratory distress attributed to sirolimus in solid organ transplant recipients. Am J Emerg Med 2015; 33: 124.e1-4.

\section{High-flow nasal oxygen for bronchoalveolar lavage in acute respiratory failure patients}

\section{To the Editor:}

Fiberoptic bronchoscopy with bronchoalveolar lavage (BAL) holds significant risks of oxygenation deterioration [1]. Among various means to improve oxygenation during BAL, noninvasive positive pressure ventilation (NPPV) has received the greatest attention [2, 3]. However, NPPV is a time-consuming and very demanding technique. High-flow nasal cannula oxygen therapy (HFNC) has emerged as a technique for noninvasive respiratory management of hypoxaemic patients [4]. In patients with acute respiratory failure (ARF), its beneficial effects have been shown in various populations [5], and its effectiveness and superiority over NPPV and conventional oxygenation recently demonstrated [6]. Its use has also been described during bronchoscopy in non-hypoxaemic [7] and in hypoxaemic patients in comparison with NPPV [8]. However, bronchoscopy was performed with an open mouth in both studies, which considerably reduces HFNC efficacy [9]. Thus, we aimed to determine HFNC's effectiveness during nasal bronchoscopy with BAL in patients with ARF along with BAL's feasibility and yield.

We conducted a prospective, observational, multicentre, open study in critically ill patients with ARF. Intensive care unit (ICU) patients from four university hospitals, with ARF and requiring a bronchoscopy with BAL were included in the study. ARF was defined as a respiratory rate $>25$ breaths $\cdot \mathrm{min}^{-1}$ (or $>20$ breaths $\mathrm{min}^{-1}$ if use of accessory respiratory muscles was present) in patients requiring oxygen at $\geqslant 6 \mathrm{~L} \cdot \mathrm{min}^{-1}$ to attain a pulse oximetry measurement of $>92 \%$. Patients were not included if they had contraindications to bronchoscopy with BAL (including respiratory acidosis).

The Ethics Committee of the French Society of Intensive Care (SRLF, Société de Réanimation de Langue Française, Paris, France) approved the study (approval number: 12-374).

HFNC was delivered via a dedicated high-flow delivery system (Optiflow; Fisher \& Paykel, Auckland, New Zealand). Oxygen flow and inspiratory oxygen fraction $\left(\mathrm{FIO}_{2}\right)$ were adjusted to obtain a pulse oximetry measurement $>92 \%$. Bronchoscopy with BAL was performed under local anaesthesia and with careful monitoring. Patients were asked to rate their dyspnoea according to a visual analogue scale, and after each BAL, the operator rated the eventual discomfort related to HFNC. Baseline and subsequent arterial blood gases were collected. Failure of the oxygenation strategy was considered if NPPV or invasive ventilation were needed within $24 \mathrm{~h}$ of the procedure. The study centres followed similar classical intubation criteria [10].

Data were compared according to failure or success of HFNC using the Mann-Whitney U-test, the paired t-test and Fisher's exact test, as appropriate. Changes in physiological measures over time were assessed using one-way ANOVA for repeated measures.

30 ICU patients (median (interquartile range (IQR)) age 54 (46-68) years) were included in the study. Demographics, physiological data and patient outcomes are detailed in table 1 . Within $24 \mathrm{~h}$, five (16.7\%) 